\title{
Relation between cyclic variation in echo amplitude and segmental contraction in normal and abnormal hearts
}

David A Lythall, Ron B Logan-Sinclair, Charles J D Ilsley, Sudhir S Kushwaha, Magdi H Yacoub, Derek G Gibson

\begin{abstract}
Objective-To study the relation between cardiac systolic activity and cardiac cycle dependent variation in the ultrasound signal arising from within the myocardium.

Design-Regional echo amplitude was used as a measure of the myocardial ultrasound signal. Relative echo amplitude values were assigned by standardising echo gain using the posterior parietal pericardium as an in-vivo calibration. $M$ mode measurements of the left ventricle were used to assess cardiac systolic activity. Subjects were studied prospectively. Analysis of echo amplitude was performed by investigators who were blinded to the results of the $M$ mode analysis. The influence of impaired left ventricular performance and abnormal wall motion were assessed.
\end{abstract}

Patients-11 cardiomyopathy patients with impaired ventricular function, eight patients with severe pulmonary hypertension and reversed septal motion, and 19 healthy controls.

Setting-All subject studies were performed at Harefield Hospital. Echo amplitude analysis was performed at the Royal Brompton Hospital.

Main outcome measures-Cyclic variation in echo amplitude was determined as the change in echo amplitude from end diastole to end systole. Additionally, an index of cyclic variation defined as the ratio of the cyclic change in echo amplitude to end diastolic echo amplitude was measured. Both cyclic variation and the cyclic variation index were analysed to see whether they correlated with left ventricular dimensions, fractional shortening, and systolic wall thickening.

Results-Stepwise regression analysis showed systolic wall thickening to be the most significant independent variable that correlated with the cyclic variation index for both the septum and posterior wall ( $r=0.68, p=0.0001$, septum; $r=$ $0.69, p=0.0001$, posterior wall). The slopes and intercepts for both regression equations were similar $(y=0.005 x+$
$0 \cdot 006$, septum; $y=0.006 x+0$, posterior wall). Subgroup analysis showed that the healthy controls, patients with cardiomyopathy, and patients with pulmonary hypertension had similar slopes and intercepts for their individual regression equations.

Conclusions-These data support the hypothesis of a quantitative relation between the extent of cyclic variation of echo amplitude and the degree of segmental myocardial shortening, as measured by systolic wall thickening, which is not significantly influenced by location within the myocardium, left ventricular performance, or wall motion. They provide further evidence of the usefulness of quantitative analysis of myocardial echo amplitude in the study of regional myocardial function in both normal and injured myocardium.

Ultrasonic tissue characterisation provides a powerful non-invasive tool for examining myocardial structure and function in both normal and disease states. ${ }^{1-10}$ Previous workers have shown a cardiac cycle dependent variation in ultrasound signals from within the myocardium in humans and animals. ${ }^{91-13}$ Peak values occurred at end diastole and minimum values at end systole. It has been suggested that these cyclic changes are related to contractile events within the myocardium. ${ }^{14}$ The extent of the relation between contractile events and cyclic variation in the ultrasound signal, however, is still unclear, particularly in human hearts both normal and diseased.

During cardiac contraction several physiological events occur within the myocardium including wall thickening, motion of the wall, and an increase in myocardial tension. Their effects on the myocardial ultrasound signal cannot easily be distinguished from each other in animal preparations-for example an increase in cardiac contractility may increase all three of these measurements, obscuring the individual contribution of each. For these reasons we have studied three different subject groups to study the effect of these physiological events on echo amplitude in humans. The three groups studied consisted of healthy volunteers in whom wall motion, thickening, 
Table 1 Clinical details for normal subjects

\begin{tabular}{|c|c|c|c|}
\hline No & Age & Sex & $\begin{array}{l}\text { Heart rate } \\
\text { (beats } / \text { min) }\end{array}$ \\
\hline 1 & 25 & $\mathbf{M}$ & 72 \\
\hline 2 & 28 & $\mathbf{M}$ & 68 \\
\hline 3 & 35 & $\mathbf{M}$ & 84 \\
\hline 4 & 21 & $\mathbf{M}$ & 60 \\
\hline 5 & 29 & $\mathbf{M}$ & 68 \\
\hline 6 & 27 & $\mathbf{M}$ & 64 \\
\hline 7 & 25 & $M$ & 60 \\
\hline 8 & 24 & $\mathbf{M}$ & 84 \\
\hline 9 & 30 & $\mathbf{M}$ & 50 \\
\hline 10 & 30 & M & 78 \\
\hline 11 & 26 & $\mathbf{M}$ & 64 \\
\hline 12 & 29 & $\mathbf{M}$ & 48 \\
\hline 13 & 34 & $\mathbf{M}$ & 52 \\
\hline 14 & 32 & $\mathbf{M}$ & 78 \\
\hline 15 & 26 & $\mathbf{M}$ & 52 \\
\hline 16 & 24 & $\mathbf{M}$ & 63 \\
\hline 17 & 27 & M & 52 \\
\hline 18 & 27 & $\mathbf{M}$ & 64 \\
\hline 19 & 26 & $M$ & 68 \\
\hline
\end{tabular}

and tension were normal; patients with cardiomyopathy in whom wall thickening and motion were decreased throughout the cycle (that is, not hypertrophic cardiomyopathy), and patients with pulmonary hypertension in whom wall thickening was normal but wall motion was abnormal. We used regional echo amplitude (echo amplitude) as a measure of the ultrasound signal arising from within the myocardium. $^{2}$

\section{Patients and methods}

We studied 19 healthy male volunteers (age 21-35 years, mean 27.6 years), 11 patients with cardiomyopathy (four female, age 21-59 years, mean 41.9 years) referred for cardiac transplant assessment, and eight patients with severe pulmonary hypertension (seven female, age 26-58 years, mean 32.6 years) referred for heart-lung transplant assessment. Table 1 shows the clinical details of the controls and table 2 of patients with cardiomyopathy or pulmonary hypertension. Two patients with cardiomyopathy were in atrial fibrillation, all other patients were in sinus rhythm. Subjects gave informed consent and the study was approved by the hospital ethics committee.

CROSS SECTIONAL AND M MODE

ECHOCARDIOGRAPHY

$M$ mode and cross sectional echocardiography were performed on a Toshiba SSH-65A echocardiograph with patients in the left lateral position. A $2.5 \mathrm{MHz}$ transducer was used. Long axis parasternal $M$ mode echograms of the left ventricle were made at the level of the mitral valve leaflet tips and were recorded on a strip chart recorder at a paper speed of $100 \mathrm{~cm} / \mathrm{s}$. Measurements were made from leading edge to leading edge and all values were the mean of five cardiac cycles. End diastolic values were measured at the onset of the $Q$ wave of the electrocardiogram and end systolic values at maximum posterior displacement of the septum except in those patients with reversed septal motion, in whom we used maximum anterior displacement of the posterior wall to define end systole. Posterior wall and septal thickening was defined as end systolic thickness minus end diastolic thickness in millimetres $(\mathrm{mm})$ and fractional shortening of the posterior wall and septum was defined as the respective change in wall thickness expressed as a percentage $(\%)$ of the diastolic dimension.

\section{ECHO AMPLITUDE STUDIES}

Echo amplitude studies were recorded with a 3.5 $\mathrm{MHz}$ Advanced Technology Laboratories (ATL) (720A/8736 series) mechanical sector scanner and an ATL (860-1 series) echocardiograph system with $45 \mathrm{~dB}$ logarithmic grey scale compression. Self correcting circuits were inactivated to avoid further differentiation of the original echo signal. Images were simultaneously displayed in grey scale and in colour by a Brompton encoder as they were recorded. Gain settings were standardised at the time the recordings were made: the master gain was increased until the parietal pericardium posterior to the left ventricle was just displayed at the highest level on the encoder (white). The depth compensation was then set to a linear ramp across the image at a rate of approximately $2 \mathrm{~dB} / \mathrm{cm}$. Gain settings were not altered thereafter. Parasternal long axis views in the left lateral position were recorded. Images were stored on Umatic $3 / 4$ inch video tape by a Sony VO 5800PS recorder. We used a Tandon PCA desk top computer for analysis. We selected stop frame end diastolic and end systolic images for analysis using a tape recorder with digital frame advance (Sony VO 5800PS). These frames were transferred from tape to a $512 \times 256 \times 6$ bit frame store. Images were processed by an adaptive filtering process to reduce speckle because this decreased echo amplitude variance and allowed smaller sample sizes to be used. ${ }^{1516}$ Areas of interest were defined in the basal ventricular septum and posterior wall corresponding to the level of the $\mathbf{M}$ mode scan and measurements of 256 individual picture elements (pixels) were made from each area. Four separate measurements each of 64 pixels were made and averaged rather than one individual

Table 2 Clinical details of patients with cardiomyopathy or pulmonary hypertension

\begin{tabular}{ccccl}
\hline No & Age & Sex & $\begin{array}{c}\text { Heart rate } \\
\text { (beats/min) Aetiology }\end{array}$ \\
\hline $\begin{array}{c}\text { Cardiomyopathy: } \\
\text { 1 }\end{array}$ & 21 & M & 94 & DCM \\
2 & 54 & M & 71 & DCM \\
3 & 23 & F & 135 & DCM \\
4 & 24 & F & 67 & RCM \\
5 & 40 & M & 69 & Sarcoid \\
6 & 51 & M & 105 & Sarcoid $\dagger$ \\
7 & 53 & M & 80 & Sarcoid \\
8 & 59 & M & 60 & DCM \\
9 & 53 & F & 122 & DCM \\
10 & 53 & M & 105 & DCM $\dagger$ \\
11 & 30 & F & 100 & DCM \\
Pulmonary hypertension: & & \\
12 & 27 & F & 71 & PPH \\
13 & 29 & F & 120 & PPH \\
14 & 26 & F & 60 & EISEN \\
15 & 28 & M & 77 & PPH \\
16 & 33 & F & 75 & PPH \\
17 & 32 & F & 87 & EISEN \\
18 & 58 & F & 83 & PH/TE \\
19 & 28 & F & 91 & EISEN \\
\hline
\end{tabular}

DCM, dilated cardiomyopathy; EISEN, Eisenmenger's syndrome; $\mathrm{PH} / \mathrm{TE}$, pulmonary hypertension secondary to thromboembolic disease; PPH, primary pulmonary hypertension; RCM, restrictive cardiomyopathy. $\star$ Abnormal septal motion; tatrial fibrillation. 
Table $3 \mathrm{M}$ mode measurements ( $\mathrm{mm}$ ) in healthy controls

\begin{tabular}{lllllllrlll}
\hline$N o$ & LVED & LVES & FS\% & $P W d$ & $P W S$ & $P W F S \%$ & $S d$ & $S s$ & $S F S \%$ & LA \\
\hline 1 & 45 & 33 & 38 & $10 \cdot 0$ & $13 \cdot 6$ & 36 & $9 \cdot 1$ & $10 \cdot 9$ & 20 & 28 \\
2 & 44 & 27 & 38 & $7 \cdot 4$ & $12 \cdot 1$ & 64 & $8 \cdot 4$ & $12 \cdot 1$ & 44 & 34 \\
3 & 51 & 34 & 34 & $8 \cdot 1$ & $13 \cdot 8$ & 69 & $6 \cdot 9$ & $13 \cdot 1$ & 91 & 38 \\
4 & 46 & 30 & 35 & $10 \cdot 0$ & $15 \cdot 0$ & 50 & $13 \cdot 3$ & $18 \cdot 8$ & 42 & 30 \\
5 & 48 & 31 & 35 & $8 \cdot 2$ & $12 \cdot 4$ & 50 & $10 \cdot 0$ & $14 \cdot 1$ & 41 & 32 \\
6 & 50 & 31 & 38 & $8 \cdot 8$ & $13 \cdot 1$ & 50 & $7 \cdot 5$ & $11 \cdot 3$ & 50 & 34 \\
7 & 49 & 30 & 37 & $8 \cdot 1$ & $16 \cdot 0$ & 97 & $10 \cdot 0$ & $12 \cdot 5$ & 25 & 38 \\
8 & 51 & 31 & 39 & $8 \cdot 8$ & $14 \cdot 1$ & 60 & $7 \cdot 1$ & $12 \cdot 9$ & 82 & 37 \\
9 & 50 & 31 & 38 & $9 \cdot 4$ & $15 \cdot 0$ & 60 & $10 \cdot 0$ & $15 \cdot 0$ & 50 & 38 \\
10 & 54 & 33 & 37 & $7 \cdot 5$ & $13 \cdot 8$ & 83 & $8 \cdot 8$ & $13 \cdot 8$ & 57 & 38 \\
11 & 53 & 34 & 36 & $10 \cdot 0$ & $15 \cdot 0$ & 50 & $8 \cdot 8$ & $13 \cdot 8$ & 57 & 30 \\
12 & 48 & 30 & 37 & $12 \cdot 5$ & $17 \cdot 5$ & 40 & $12 \cdot 5$ & $17 \cdot 5$ & 40 & 41 \\
13 & 45 & 29 & 36 & $10 \cdot 1$ & $15 \cdot 4$ & 52 & $8 \cdot 7$ & $15 \cdot 1$ & 74 & 33 \\
14 & 46 & 33 & 29 & $7 \cdot 5$ & $13 \cdot 1$ & 73 & $9 \cdot 3$ & $12 \cdot 5$ & 34 & 44 \\
15 & 48 & 31 & 35 & $10 \cdot 7$ & $15 \cdot 4$ & 44 & $9 \cdot 3$ & $12 \cdot 4$ & 33 & 39 \\
16 & 39 & 23 & 40 & $7 \cdot 1$ & $10 \cdot 3$ & 45 & $7 \cdot 6$ & $10 \cdot 9$ & 43 & 20 \\
17 & 47 & 29 & 39 & $9 \cdot 9$ & $14 \cdot 9$ & 51 & $10 \cdot 0$ & $13 \cdot 8$ & 38 & 30 \\
18 & 49 & 31 & 37 & $7 \cdot 3$ & $12 \cdot 4$ & 69 & $7 \cdot 2$ & $12 \cdot 0$ & 63 & 32 \\
19 & 52 & 38 & 28 & $8 \cdot 3$ & $14 \cdot 6$ & 77 & $10 \cdot 6$ & $17 \cdot 5$ & 65 \\
Mean (SD) & $48(3 \cdot 4)$ & $31(2 \cdot 9)$ & $36(3 \cdot 2)$ & $8 \cdot 9(1 \cdot 4)$ & $14 \cdot 1(1 \cdot 6)$ & $59(15 \cdot 9)$ & $9 \cdot 2(1 \cdot 7)$ & $13 \cdot 7(2 \cdot 3)$ & $50(18 \cdot 7)$ & $35(5 \cdot 6)$ \\
\end{tabular}

FS\%, percentage fractional shortening of left ventricle; LA, left atrial dimension; LVED, left ventricular end diastolic dimension LVES, left ventricular end systolic dimension; PWd, Posterior wall end diastolic thickness; PWFS\%, percentage posterior wal systolic thickening; PWs, posterior wall end systolic thickness; Sd, septal end diastolic thickness; SFS \%, percentage septal systolic thickening; Ss, septal end systolic thickness.

measurement of 256 pixels because this allowed the pixel matrix to be placed within the mid-myocardium and thus avoided including specular echoes from the endocardium or epicardium. A maximum pixel intensity for each image was derived by measuring the maximum colour intensity of the superimposed colour scale. This corresponded to a value of $45 \mathrm{~dB}$ thus allowing echo amplitude values to be assigned. $A$ value of $0 \mathrm{~dB}$ would therefore correspond to a level $-45 \mathrm{~dB}$ below the parietal pericardial echo amplitude. Individual pixel amplitudes were displayed as a histogram and a separate digital readout displayed mean, median, and standard deviation values for each region measured. In addition to values of echo amplitude at end diastole and end systole, an index of the change in echo amplitude during the cardiac cycle (cyclic variation index) was calculated as the ratio of end diastolic echo amplitude minus end systolic echo amplitude to end diastolic echo amplitude.
STATISTICAL ANALYSIS

All values are expressed as mean (SD). We used stepwise multivariate and univariate linear regression analysis to analyse the relation between echo amplitude and $M$ mode measurements, age, and heart rate. Intergroup comparisons were made by two tailed $t$ testing. A probability level of $p<0.05$ was regarded as significant.

\section{Results}

Images suitable for analysis were available in all but one control in whom an adequate end systolic image of the septum could not be obtained and in two patients with severe pulmonary hypertension in whom adequate views of the septum could not be obtained in either systole or diastole. In one patient with pulmonary hypertension left atrial dimensions could not be measured reliably. Table 3 shows the $M$ mode values for individual subjects.

Table $4 \mathrm{M}$ mode measurements $(\mathrm{mm})$ in patients with cardiomyopathy or pulmonary hypertension

\begin{tabular}{|c|c|c|c|c|c|c|c|c|c|c|}
\hline No & LVED & LVES & $F S \%$ & $P W d$ & PWs & $P W F S \%$ & $S d$ & Ss & $S F S \%$ & $L A$ \\
\hline \multicolumn{11}{|c|}{ Cardiomyopathy: } \\
\hline 1 & 70 & 64 & 8 & $7 \cdot 2$ & $8 \cdot 3$ & 15 & $7 \cdot 2$ & $7 \cdot 8$ & 8 & 46 \\
\hline 2 & 80 & 73 & 9 & $6 \cdot \overline{6}$ & 7.9 & 19 & $6 \cdot \overline{6}$ & 7.9 & 19 & 76 \\
\hline 3 & 55 & 51 & 7 & $11 \cdot 4$ & $12 \cdot 9$ & 13 & $10 \cdot 0$ & $11 \cdot 4$ & 14 & 36 \\
\hline 4 & 38 & 29 & 24 & $10 \cdot 0$ & $13 \cdot 0$ & 30 & $10 \cdot 5$ & $13 \cdot 0$ & 24 & 46 \\
\hline 5 & 56 & 46 & 18 & $9 \cdot 0$ & 14.5 & 61 & $14 \cdot 5$ & $18 \cdot 0$ & 24 & 45 \\
\hline 6 & 29 & 22 & 24 & $7 \cdot 0$ & $11 \cdot 0$ & 57 & $9 \cdot 0$ & $12 \cdot 0$ & 33 & 41 \\
\hline 7 & 102 & 94 & 8 & $5 \cdot 5$ & 6.0 & 9 & $5 \cdot 0$ & $7 \cdot 0$ & 30 & 32 \\
\hline 8 & 64 & 44 & 31 & 8.5 & $14 \cdot 0$ & 65 & $8 \cdot 5$ & $13 \cdot 0$ & 53 & 42 \\
\hline 9 & 59 & 55 & 7 & $5 \cdot 5$ & 6.0 & 9 & $6 \cdot 0$ & 6.5 & 8 & 37 \\
\hline 10 & 82 & 67 & 18 & $11 \cdot 0$ & $13 \cdot 0$ & 18 & $11 \cdot 0$ & $12 \cdot 0$ & 9 & 59 \\
\hline 11 & 56 & & & $9 \cdot 5$ & $10 \cdot 5$ & 11 & $7 \cdot 0$ & $7 \cdot 3$ & 4 & 39 \\
\hline Mean (SD) & $63(20 \cdot 4)$ & $54(20 \cdot 1)$ & $14(8.9)$ & $8 \cdot 3(2 \cdot 1)$ & $10 \cdot 6(3 \cdot 1)$ & $28(22 \cdot 2)$ & $8 \cdot 7(2 \cdot 7)$ & $10.5(3.6)$ & $21(14 \cdot 5)$ & $45(12 \cdot 4)$ \\
\hline \multicolumn{11}{|c|}{ Pulmonary hypertension: } \\
\hline 12 & 28 & 10 & 29 & $8 \cdot 0$ & $11 \cdot 0$ & 38 & $9 \cdot 0$ & $15 \cdot 0$ & 67 & 19 \\
\hline 13 & 38 & 11 & 71 & $7 \cdot 0$ & 10.0 & 42 & $5 \cdot 5$ & $7 \cdot 5$ & 36 & 36 \\
\hline 14 & 55 & 27 & 51 & 10.0 & 14.5 & 45 & $10 \cdot 5$ & $15 \cdot 0$ & 43 & $\star$ \\
\hline 15 & 41 & 32 & 22 & 8.5 & 13.0 & 53 & $10 \cdot 5$ & 14.0 & 33 & 30 \\
\hline 16 & 27 & 13 & 59 & $9 \cdot 0$ & 12.0 & 33 & 8.5 & 12.0 & 41 & 28 \\
\hline 17 & 32 & 23 & 28 & 6.0 & $8 \cdot 5$ & 42 & $6 \cdot 0$ & $7 \cdot 0$ & 13 & 31 \\
\hline 18 & 25 & 12 & 52 & 7.0 & $10 \cdot 0$ & 43 & $7 \cdot 3$ & $9 \cdot 0$ & 23 & 26 \\
\hline 19 & 35 & 26 & 26 & $8 \cdot 0$ & $11 \cdot 0$ & 38 & $7 \cdot 0$ & 8.5 & 21 & 29 \\
\hline Mean (SD) & $35(9 \cdot 8)$ & $19(8 \cdot 7)$ & $42(18 \cdot 3)$ & $7.9(1.3)$ & $11.3(1.9)$ & $42(5.9)$ & $8.0(1.9)$ & $11 \cdot 0(3 \cdot 4)$ & $35(16 \cdot 6)$ & $28(5 \cdot 2)$ \\
\hline
\end{tabular}

*Could not be measured.

See table 3 for all other abbreviations. 


\section{VENTRICULAR SEPTAL MOTION}

All the patients with pulmonary hypertension had reversed ventricular septal motion with considerable bowing of the interventricular septum into the left ventricle. Three patients in the cardiomyopathy group also had abnormal septal motion: two patients because of left bundle branch block and one associated with severe pulmonary hypertension. All the other subjects had normal septal motion.

\section{LEFT VENTRICULAR AND LEFT ATRIAL}

\section{DIMENSIONS (TABLES 3 AND 4)}

Left ventricular end diastolic dimension and end systolic dimension were greater $(p<$ $0.005)$ and percentage fractional shortening was less $(p<0.001)$ in the patients with cardiomyopathy than in the healthy controls, reflecting the poor left ventricular systolic function of the cardiomyopathy patients. Left ventricular dimensions in the patients with pulmonary hypertension were significantly lower than for the healthy controls $(p<0.001)$ although fractional shortening was maintained reflecting the apparent distortion of left ventricular shape and the smaller body mass of these patients (seven of the eight patients were female). The right ventricular cavity was considerably enlarged and the right ventricular free wall appeared to be thickened in the patients with pulmonary hypertension.

\section{WALL THICKENING}

Tables 3 and 4 show the values for posterior wall and septal thickness. The mean end systolic wall thickness of the posterior wall and septum was significantly lower in the cardiomyopathy patients than in the healthy controls $(\mathrm{p}<0.01)$, again reflecting the grossly impaired contractile function of these patients' hearts. Similarly, percentage systolic thickening of both the posterior wall and the septum was significantly lower $(p<0.0001)$. Systolic wall thickening was preserved in the patients with pulmonary hypertension and reversed septal motion. For both the controls and the patients with pulmonary hypertension, wall thickening was within the normal range reported by others. ${ }^{17}$

\section{REGIONAL ECHO AMPLITUDE (TABLES 5 AND 6)}

The mean echo amplitude in the septum of the healthy controls was significantly greater at end diastole than end systole ( $p=0.0001)$. A similar variation during the cardiac cycle was seen in the posterior wall $(p=0.0001)$. Figure 1 shows representative end diastolic and end systolic echo amplitude images from a healthy volunteer. In the patients with pulmonary hypertension we saw variation in echo amplitude of a similar magnitude, greatest at end diastole and least at end systole, in the septum $(p<0.05)$ and posterior wall $(p<$ 0.001 ). In contrast, the variation in echo amplitude during the cardiac cycle was considerably blunted in the poorly contractile left ventricles of the patients with cardiomyopathy. In three patients with cardiomyopathy with greatly reduced posterior wall thickening and in one with very reduced septal thickening (cardiomyopathy patients 3, 7, 9 and 11 respectively) echo amplitude at end systole was slightly higher than at end diastole. The index of echo amplitude cyclic variation was less in the patients with cardiomyopathy than in the healthy controls (septum, $\mathrm{p}=0.09$; posterior wall, $p<0.001)$ and in the patients with the pulmonary hypertension (septum, $p=0.01$; posterior wall, $\mathrm{p}=0.001$ )

\section{ECHO AMPLITUDE CYCLIC VARIATION VERSUS} SYSTOLIC WALL THICKENING

Stepwise linear regression analysis showed that systolic wall thickening was the most important independent determinant of echo amplitude cyclic variation, measured as the cyclic variation index (table 7). The slopes and intercepts of the regression relation for both the septum (y $=0.005 \mathrm{x}+0.06)$ and posterior wall $(\mathrm{y}=$ $0.006 x+0.004$ ) were similar (fig 2), suggesting that at least in the base of the left ventricle the relation between cyclic variation in echo

Table 5 Regional echo amplitude values in healthy controls

\begin{tabular}{|c|c|c|c|c|c|c|}
\hline No & $\begin{array}{l}S E A d \\
(d B)\end{array}$ & $\begin{array}{l}S E A s \\
(d B)\end{array}$ & $S C V I$ & $\begin{array}{l}P W E A d \\
(d B)\end{array}$ & $\begin{array}{l}P W E A s \\
(d B)\end{array}$ & $P W C V I$ \\
\hline $\begin{array}{l}1 \\
2 \\
3 \\
4 \\
5 \\
6 \\
7 \\
8 \\
9 \\
10 \\
11 \\
12 \\
13 \\
14 \\
15 \\
16 \\
17 \\
18 \\
19 \\
\text { Mean (SD) }\end{array}$ & $\begin{array}{l}2.79 \\
2.83 \\
4.03 \\
2.48 \\
5 \cdot 15 \\
3.43 \\
3.01 \\
2.83 \\
3.13 \\
3.90 \\
1.77 \\
2.45 \\
4.65 \\
3.65 \\
3.56 \\
4.54 \\
2.98 \\
3.43 \\
4.02 \\
3.40(0.85)\end{array}$ & $\begin{array}{l}2 \cdot 58 \\
2 \cdot 44 \\
2 \cdot 10 \\
2 \cdot 15 \\
4 \cdot 18 \\
2.62 \\
2.87 \\
2 \cdot 13 \\
1.77 \\
2.91 \\
\star \\
1.90 \\
2.76 \\
2.81 \\
2.66 \\
3.29 \\
2.50 \\
2.47 \\
1.83 \\
2.55(0.58)\end{array}$ & $\begin{array}{l}0.07 \\
0.14 \\
0.48 \\
0.13 \\
0.19 \\
0.24 \\
0.05 \\
0.25 \\
0.43 \\
0 \cdot 25 \\
\star \\
0 \cdot 23 \\
0.41 \\
0.23 \\
0.25 \\
0.28 \\
0 \cdot 16 \\
0.28 \\
0.54 \\
0.26(0.14)\end{array}$ & $\begin{array}{l}3.63 \\
4 \cdot 03 \\
4 \cdot 43 \\
5 \cdot 70 \\
5 \cdot 35 \\
3.53 \\
5.09 \\
3 \cdot 12 \\
2 \cdot 25 \\
4.09 \\
4.32 \\
2.96 \\
3.45 \\
4 \cdot 10 \\
4 \cdot 12 \\
3.05 \\
4 \cdot 18 \\
3.81 \\
4.06 \\
3.96(0.84)\end{array}$ & $\begin{array}{l}3.57 \\
2 \cdot 16 . \\
2.98 \\
3.16 \\
4.40 \\
2.25 \\
2.74 \\
2.44 \\
1.89 \\
2.58 \\
3.20 \\
2.61 \\
1.96 \\
2.44 \\
2.41 \\
2.59 \\
2.19 \\
2.37 \\
2.05 \\
2.63(0.62)\end{array}$ & $\begin{array}{l}0.02 \\
0.46 \\
0.33 \\
0.45 \\
0.18 \\
0.36 \\
0.46 \\
0.22 \\
0 \cdot 16 \\
0.37 \\
0.26 \\
0.12 \\
0.43 \\
0.40 \\
0.41 \\
0.15 \\
0.48 \\
0.38 \\
0.50 \\
0.32(0.14)\end{array}$ \\
\hline
\end{tabular}

PWEAd, posterior wall end diastolic echo amplitude (dB); PWEAs, posterior wall end systolic echo amplitude (dB); PWCVI, posterior wall cyclic variation index (PWEAd - PWEAs) $\div$ PWEAd); SEAd, septal end diastolic echo amplitude (dB); SEAs, poptal end systolic echo amplitude (dB); SCVA, septal cyclic variation index (SEAd - SEAs) $\div$ SEAd).

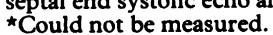


Table 6 Regional echo amplitude in patients with cardiomyopathy or pulmonary hypertension

\begin{tabular}{|c|c|c|c|c|c|c|}
\hline No & $\begin{array}{l}S E A d \\
(d B)\end{array}$ & $\begin{array}{l}S E A s \\
(d B)\end{array}$ & $S C V I$ & $\begin{array}{l}\text { PWEAd } \\
(d B)\end{array}$ & $\begin{array}{l}P W E A s \\
(d B)\end{array}$ & $P W C V I$ \\
\hline \multicolumn{7}{|c|}{ Cardiomyopathy: } \\
\hline 1 & $7 \cdot 48$ & $5 \cdot 73$ & 0.23 & $14 \cdot 70$ & $12 \cdot 38$ & $0 \cdot 16$ \\
\hline 2 & $7 \cdot 88$ & $7 \cdot 71$ & 0.02 & 8.68 & $7 \cdot 86$ & 0.09 \\
\hline 3 & $8 \cdot 31$ & 7.95 & 0.04 & $9 \cdot 83$ & $10 \cdot 07$ & -0.02 \\
\hline 4 & $6 \cdot 56$ & $5 \cdot 23$ & 0.20 & $7 \cdot 01$ & $4 \cdot 77$ & 0.32 \\
\hline 5 & 8.05 & $6 \cdot 42$ & $0 \cdot 20$ & $7 \cdot 29$ & $6 \cdot 89$ & 0.06 \\
\hline 6 & $6 \cdot 82$ & 3.64 & 0.47 & $7 \cdot 24$ & $5 \cdot 43$ & 0.25 \\
\hline 7 & $7 \cdot 24$ & 5.90 & $0 \cdot 19$ & $7 \cdot 18$ & $7 \cdot 76$ & -0.08 \\
\hline 8 & $5 \cdot 23$ & $3 \cdot 68$ & 0.30 & $8 \cdot 52$ & $5 \cdot 82$ & 0.32 \\
\hline 9 & 8.97 & 7.94 & $0 \cdot 12$ & $12 \cdot 28$ & $13 \cdot 73$ & $-0 \cdot 12$ \\
\hline 10 & 7.86 & $7 \cdot 32$ & 0.07 & $8 \cdot 38$ & $7 \cdot 85$ & 0.06 \\
\hline 11 & $10 \cdot 74$ & $11 \cdot 19$ & -0.04 & $8 \cdot 37$ & 7.98 & 0.05 \\
\hline Mean (SD) & $7.74(1.41)$ & $6 \cdot 61(2 \cdot 17)$ & $0 \cdot 16(0 \cdot 14)$ & $9 \cdot 04(2.41)$ & $8 \cdot 32(2 \cdot 81)$ & $0 \cdot 10(0 \cdot 15)$ \\
\hline \multicolumn{7}{|c|}{ Pulmonary hypertension: } \\
\hline 12 & 8.82 & 3.97 & 0.55 & $6 \cdot 05$ & $4 \cdot 20$ & 0.31 \\
\hline 13 & $4 \cdot 73$ & 2.63 & 0.44 & $3 \cdot 50$ & $2 \cdot 33$ & 0.33 \\
\hline 14 & 2.66 & $1 \cdot 76$ & 0.34 & 5.53 & 3.05 & 0.45 \\
\hline 15 & $3 \cdot 61$ & $2 \cdot 50$ & 0.31 & $4 \cdot 28$ & $2 \cdot 00$ & 0.53 \\
\hline 16 & 4.52 & $2 \cdot 47$ & 0.45 & $4 \cdot 35$ & $3 \cdot 26$ & 0.25 \\
\hline 17 & $2 \cdot 18$ & 1.87 & $0 \cdot 14$ & 4.03 & $3 \cdot 18$ & 0.21 \\
\hline 18 & $\star$ & $\star$ & $\star$ & 8.99 & $5 \cdot 86$ & 0.35 \\
\hline 19 & $\star$ & $\star$ & $\star$ & $9 \cdot 84$ & $7 \cdot 26$ & $0 \cdot 26$ \\
\hline $\operatorname{Mean}(\mathrm{SD})$ & $4.42(2 \cdot 38)$ & $2.55(0.79)$ & $0.37(0.14)$ & $5 \cdot 82(2 \cdot 37)$ & $3.89(1.81)$ & $0.34(0.11)$ \\
\hline
\end{tabular}

See footnote to table 5 for abbreviations.

amplitude and systolic wall thickening is independent of position within the ventricle and mainly reflects the degree of wall thickening. Analysis of the three groups individually confirmed a similar relation within each group. The slopes and intercepts of the regression relation were similar in the healthy controls and patients with cardiomyopathy but there was some variation in these values in the patients with pulmonary hypertension (figs 3 and 4). However, there was a large overlap in the $95 \%$ confidence intervals for the slopes between the three groups. The correlation between the absolute change in echo amplitude (as distinct from cyclic variation index) and systolic wall thickening was less $(\mathrm{r}=0.42, \mathrm{p}=0.01$, septum all subjects; $r=0.47, p=0.003$, posterior wall all subjects), suggesting that the incorporation of a factor, in this case end diastolic echo amplitude, which reflects the overall background level of echo amplitude within the myocardium, is important.

ECHO AMPLITUDE CYCLIC VARIATION VERSUS CAVITY DIMENSION, WALL THICKNESS, HEART RATE, AND AGE

Systolic but not diastolic wall thickness was significantly correlated with the cyclic variation index for both the septum $(r=0.36, p=0.02)$ and posterior wall $(r=0.50, p=0.002)$ in all subjects. The left ventricular end diastolic dimension was significantly negatively correlated with the cyclic variation index, both for the septum $(r=-0.42, p=0.02)$ and posterior wall $(\mathrm{r}=-0.45, \mathrm{p}=0.005)$ as was left ventricular end systolic dimension (septum, $r=-0.51 ; p=0.002:$ posterior wall, $r=-0.58 ; p=0.0001)$. Left ventricular fractional shortening was also significantly correlated with cyclic variation index (septum, $r=0.48 ; p=0.004$ : posterior wall, $r=0.53$; $p=0.001)$. These results are consistent with the major influence of systolic wall thickening on overall left ventricular performance. Heart rate and age were negatively correlated with the cyclic variation index in the posterior wall (heart rate, $r=-0.48 ; p=0.003$ : age, $r$ $=-0.35 ; \mathrm{p}=0.03$ ) but no similar correlation was seen for the septum. The negative correlation with heart rate seemed to reflect the strong association between poor left ventricular function and the higher heart rates observed clinically.

\section{REPRODUCIBILITY OF ECHO AMPLITUDE} MEASUREMENTS

To measure the reproducibility of this technique, 30 end diastolic $(3 \cdot 28-7 \cdot 84 \mathrm{~dB})$ and 30 end systolic (2.96-4.88 dB) measurements were analysed on two separate occasions by the same observer (DL). The mean difference between repeated end diastolic measurements was -0.05 (SD 0.58) dB. For end systolic measurements the mean difference was -0.02 (SD 0.60) dB.

\section{Discussion}

We found a quantitative relation between cyclic variation in echo amplitude and segmental contractile events, as reflected by systolic wall thickening, within the myocardium of human subjects. This relation seemed to be valid over a wide range of both physiological and pathological degrees of left ventricular performance, even in the presence of distorted wall motion as illustrated by those patients with severe pulmonary hypertension and reversed septal motion. It seemed to be relatively independent of alterations in wall tension-in that myocardium with presumed increased levels of wall tension (dilated cardiomyopathies) behaved similarly to myocardium with normal values (controls) and probably also to myocardium with decreased values (left ventricular posterior wall in reversed septal motion). Similarly, local geometric factors such as diastolic wall thickness had little influence. Global left ventricular characteristics such as left ventricular dimension and fractional shor- 


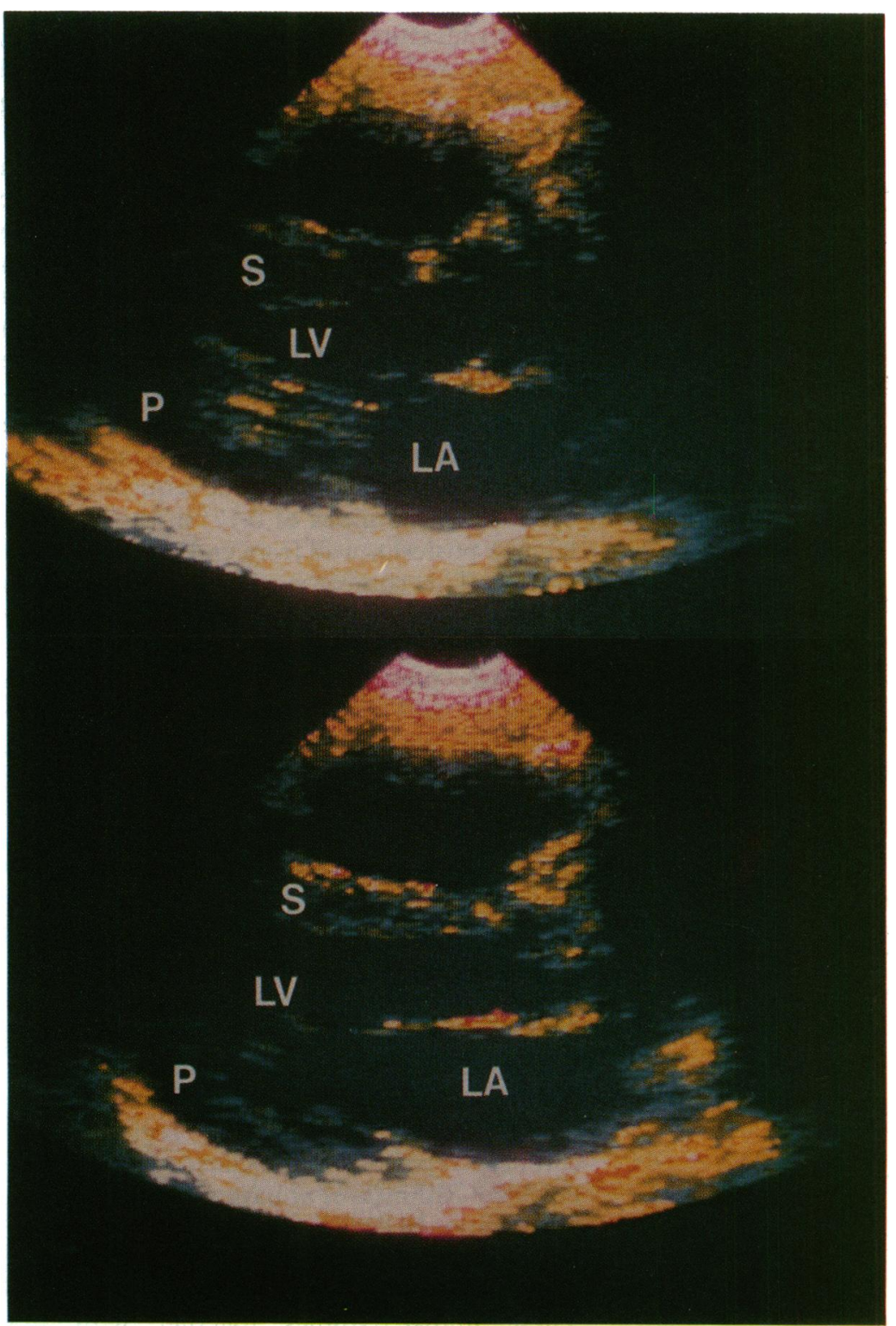

Figure 1 Representative colour-encoded images from a healthy control. (Top) End systolic frame and (bottom) preceding end diastolic frame. Dark blue represents the lowest levels of echo amplitude, which progresses through green, yellow, red, and purple to white, the highest level of echo amplitude. The myocardium of the posterior wall and interventricular septum at end diastole is predominantly green-blue. The amplitude intensity of echoes from the same areas of myocardium at end systole (predominantly dark blue) was less than at end diastole. The higher amplitude echoes seen anterior to (above) the posterior wall in the end systolic image are related to the mitral valve apparatus. $S$, interventricular septum; $L A$, left atrium; $L V$, left ventricular cavity; $P$, posterior wall.
PREVIOUS HUMAN AND ANIMAL STUDIES

There is considerable evidence for a causal relation between cyclic variation and cardiac systolic activity. In animal models it has been shown that interventions that depress myocardial function, such as ischaemia, cause a blunting of cyclic variation in the myocardial ultrasound signal, in this case integrated backscatter. ${ }^{13}$ Wickline et al used paired pacing and propranolol to modify myocardial contractility in open chested dogs and they showed that the maximum negative rate of change of integrated backscatter $\left(-\mathrm{dIB} / \mathrm{dt}_{\max }\right)$ seemed to change in parallel with global contractile function measured by the maximum rate of change of left ventricular pressure $\left(\mathrm{dP} / \mathrm{dt}_{\max }\right){ }^{18}$ However, they were unable to correlate the extent of cyclic variation of integrated backscatter with changes in left ventricular $\mathrm{dP} / \mathrm{dt}_{\max }$. They did show a regional variation within the left ventricle, the amplitude of cyclic variation being greater in the apical than the basal regions of the ventricle and they suggested that cyclic variation in integrated backscatter may in part be dependent on absolute sarcomere shortening which may be greater in more apical regions of the ventricle. Glueck et al also showed a progressive increase in cyclic variation from ventricular base to apex which they suggested paralleled a similar gradation in contractile function. ${ }^{19}$ In later experiments Wickline $e t$ al, again in open chested dogs, showed a significant correlation between another measure of cyclic variation (phase weighted amplitude of cyclic variation) and systolic wall thickening before and during coronary occlusion, and after reperfusion. ${ }^{20}$ Below $20 \%$ systolic thickening this relation was markedly non-linear with large decrements in phase weighted amplitude occurring with small decreases in systolic thickening. Non-linearity at non-physiological levels of systolic thickening may reflect irreversible or slowly reversible structural or biochemical alterations within the myocardium, since it has previously been shown that the collagen matrix is damaged after even brief periods of ischaemia. ${ }^{21}$ These findings are similar to our observations at the very low levels of wall thickening seen in the cardiomyopathy study group, perhaps reflecting the irreversible changes in myocardial structure that occur in end-stage cardiomyopathy. Sagar et al studied the effect of heart rate, preload, and inotropic state on cyclic variation and also found a relation with grouped data between cyclic variation and segmental shortening

Table 7 Univariate regression analysis of echo amplitude cyclic variation index compared with percentage systolic thickening

\begin{tabular}{|c|c|c|c|c|c|c|c|c|}
\hline & $n$ & $r$ & $r^{2}$ & $S E$ & $p$ & Intercept & Slope & SE slope \\
\hline \multicolumn{9}{|l|}{ Septum: } \\
\hline All subjects & 35 & 0.68 & 0.46 & $0 \cdot 114$ & 0.0001 & 0.06 & 0.005 & 0.001 \\
\hline Controls & 18 & 0.73 & 0.53 & 0.096 & 0.0006 & 0.00 & 0.005 & 0.001 \\
\hline Cardiomyopathy & 11 & 0.67 & 0.45 & $0 \cdot 113$ & 0.025 & 0.03 & 0.007 & 0.002 \\
\hline PHT & 6 & 0.90 & 0.81 & 0.07 & 0.015 & 0.09 & 0.007 & 0.002 \\
\hline \multicolumn{9}{|l|}{ Posterior wall: } \\
\hline All subjects & 38 & 0.69 & 0.47 & $0 \cdot 126$ & 0.0001 & 0.00 & 0.006 & 0.001 \\
\hline Controls & 19 & 0.54 & $0 \cdot 29$ & 0.124 & 0.018 & 0.04 & 0.005 & 0.001 \\
\hline Cardiomyopathy & 11 & 0.65 & 0.42 & $0 \cdot 120$ & 0.03 & -0.02 & 0.004 & 0.002 \\
\hline PHT & 8 & 0.83 & 0.69 & 0.065 & 0.01 & $-0 \cdot 29$ & 0.015 & 0.004 \\
\hline
\end{tabular}

$\mathrm{n}$, number; $\mathrm{p}$, probability; PHT, pulmonary hypertension; r, correlation coefficient; SE, standard error. 


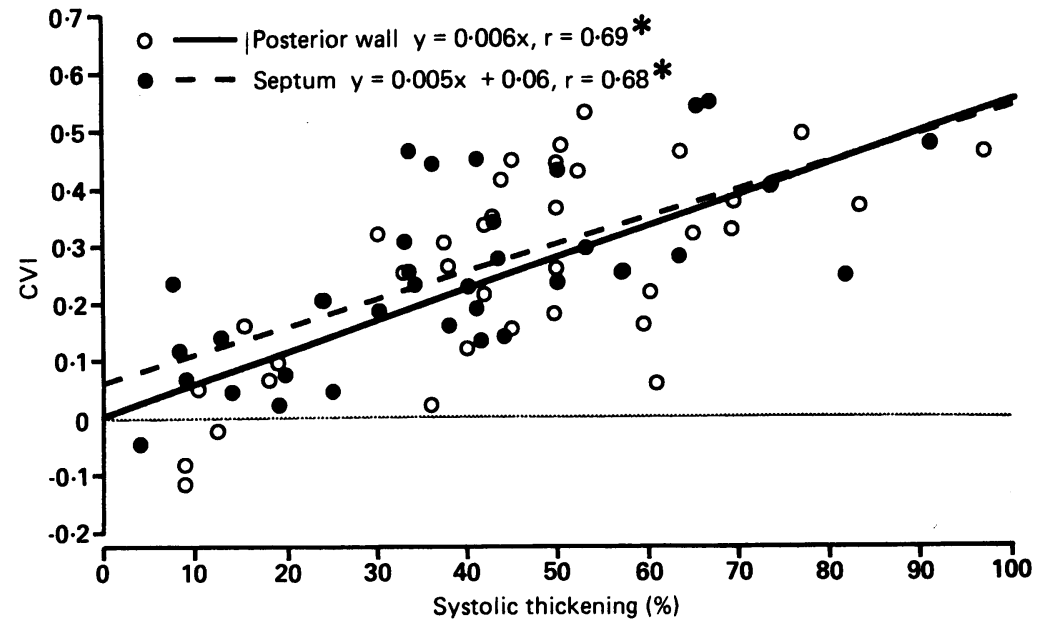

Figure 2 Cyclic variation index plotted against percentage systolic wall thickening for both the septum and posterior wall for all subjects. The slopes and intercepts for both relations are similar, suggesting that this relation is relatively independent of position within the ventricular myocardium. Cyclic variation index $(C V I)=($ end diastolic $E A$ - end systolic $E A) \div$ end diastolic $E A{ }^{\star}{ }^{\star} p=0.0001$.

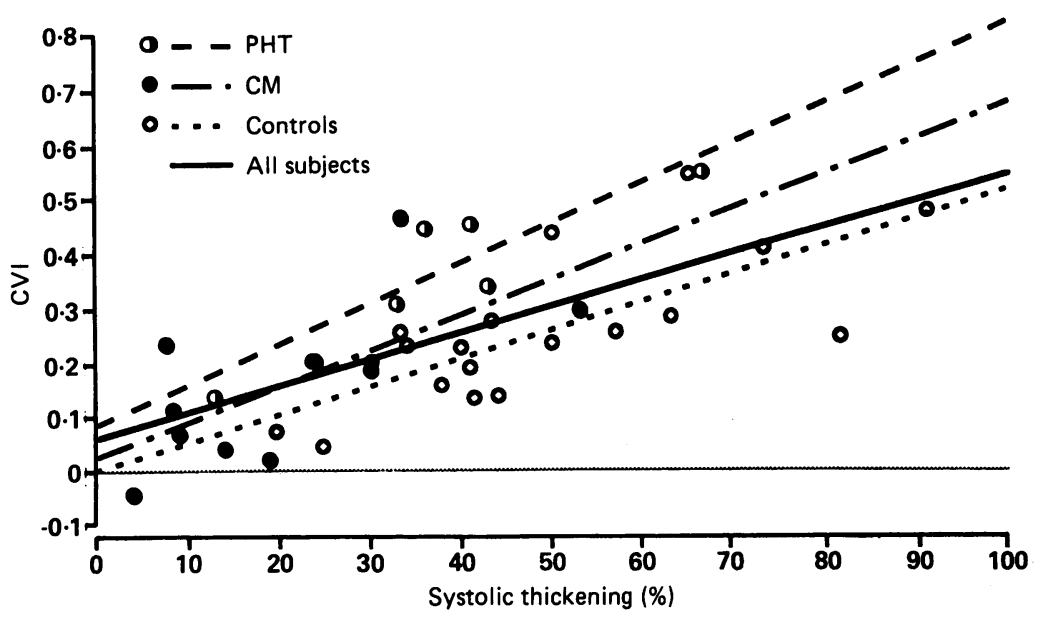

Figure 3 Cyclic variation index plotted against percentage systolic wall thickening for the septum for all three groups. The slopes and intercepts for the series of regressions are similar, suggesting that the relation is independent of left ventricular performance and is not altered in the presence of abnormal wall motion. CM, cardiomyopathy;

PHT, pulmonary hypertension. See legend to figure 2 for other abbreviations.

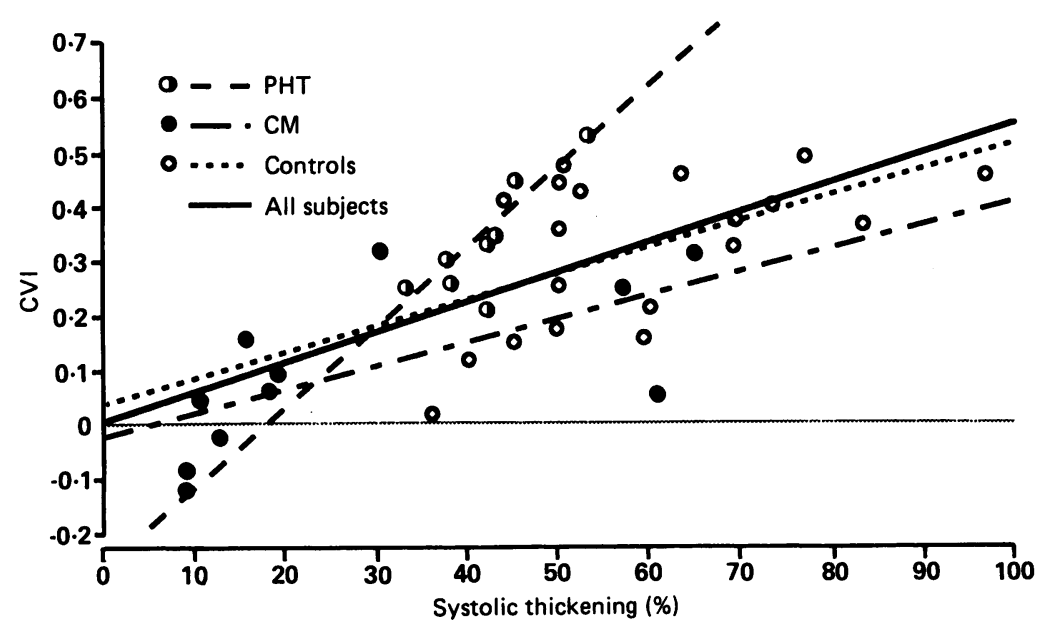

Figure 4 Cyclic variation index plotted against percentage systolic wall thickening for the posterior wall for all three groups. Slopes and intercepts for the series of regressions for controls and patients with cardiomyopathy are similar suggesting that the relation is independent of left ventricular performance. There is some variance with the patients with pulmonary hypertension; however, there is overlap between the $95 \%$ confidence intervals for the slopes of all three regression lines. CM, cardiomyopathy; $P H T$ pulmonary hypertension. See legend to figure 2 for other abbreviations. directly measured by sonomicrometry and with left ventricular $\mathrm{dP} / \mathrm{dt}_{\max } \cdot{ }^{22}$ However, they did not directly correlate segmental shortening with cyclic variation.

Human studies have also suggested a relation between cyclic variation and cardiac systolic activity. Olshansky et al analysed regional echo amplitude, using the mean grey level, from cross sectional echocardiograms in 16 healthy controls and confirmed a cyclic variation in echo amplitude. ${ }^{12}$ In six subjects digitised stop frame photographs were analysed and in the remaining 10 data were acquired directly from the digital scan converter. They showed an average fall of $17 \%$ in echo amplitude (equivalent to a cyclic variation index of 0.17 ) in the left ventricular posterior wall from end diastole to end systole. This compares with an average cyclic variation index in healthy controls in this study of $0.32(0 \cdot 14)$. We believe this difference reflects the lesser pre-processing of our equipment. Vered et $a l^{9}$ using integrated backscatter measurements also showed cyclic variation in normal human myocardium. ${ }^{9}$ They also saw considerably diminished cyclic variation in five patients with dilated cardiomyopathy. Similarly, a study of integrated backscatter in patients at the time of coronary angioplasty showed a blunting of cyclic variation associated with wall motion abnormalities during balloon inflation with a return to baseline after balloon deflation. $^{23}$ More recently Milunski and colleagues showed a recovery in cyclic variation, measured using integrated backscatter, in infarcted and peri-infarcted regions after coronary reperfusion associated with thrombolysis. ${ }^{24}$ Milunski et al have also reported the preservation of cyclic variation in patients with reversed septal motion ${ }^{25}$ similar to our findings in patients with pulmonary hypertension. These human studies together with the previous animal experiments indicate a correlation between cyclic variation in the myocardial ultrasound signal and myocardial systolic activity.

The exact basis of this association is as yet unclear. Because systolic thickening is directly related to myocyte shortening, since the shortening myocardial segment must thicken for its volume to remain constant, it is likely that the mechanism that produces cyclic variation in echo amplitude is substantially related to myocyte shortening. It has been suggested that as myocytes contract the acoustic properties of the contractile and elastic elements may alter in disparate directions thus changing the acoustic mismatch and causing a fall in the reflected ultrasound signal. ${ }^{14}$ The cyclic variation in the ultrasound signal could thus be modified to parallel contractile events in timing as well as in magnitude. During myocardial contraction myocardial fibre orientation changes ${ }^{26}$ and this is also likely to influence the ultrasound signal. The relative uniformity of echo amplitude cyclic variation seen in this study at the base of the heart, in both the septum and posterior wall, as evidenced by the similar slopes and intercepts of the cyclic variation index versus systolic thickening relation, may reflect the similar arrangement of circumferential and 
longitudinal fibres at this level ${ }^{27} \mathrm{By}$ comparison the increased cyclic variation in distal regions of the canine left ventricle seen by Glueck et al ${ }^{19}$ may reflect the higher proportion of longitudinal fibres and increased wall torsion at the apex. ${ }^{28}$ Again relative fibre rearrangement within the myocardium during the cardiac cycle will influence the degree of wall thickening. Although the exact relation between myocardial systolic activity and cyclic variation is unclear, it is likely that both segmental myocardial contraction as evidenced by systolic thickening and cyclic variation in the ultrasound signal reflect conformational changes in contractile elements within the myocardium at either or both cellular and fibre levels.

Several workers who used integrated backscatter measurements for examining cyclic variation in the myocardial ultrasound signal have noted a disparity between systolic wall thickening and cyclic variation in both myocardial ischaemia and hypertrophy..$^{2029}$ In their studies cyclic variation was determined as absolute change and did not incorporate any measure of background levels of ultrasound signal. Our use of the parietal pericardium as a method for standardising echo amplitude allows values of echo amplitude at end diastole and end systole to be assigned and enables the ratio of change in echo amplitude (cyclic variation index) to be calculated. Our results showed a considerable and significant improvement in correlation when the cyclic variation index rather than absolute change in echo amplitude was compared with systolic thickening. This suggests for example that a cyclic variation of $2 \mathrm{~dB}$ at a background end diastolic echo amplitude of $4 \mathrm{~dB}$ is associated with a greater degree of systolic wall thickening than a similar change of $2 \mathrm{~dB}$ at an end diastolic echo amplitude of $10 \mathrm{~dB}$. We have previously shown that echo amplitude is increased in both left ventricular hypertrophy and ischaemic heart disease $^{56}$ and is associated with increased myocardial collagen in ischaemic heart disease and abnormalities of diastolic function such as diminished peak rate of wall thinning and cavity dimension increase in left ventricular hypertrophy. It is also known that the amplitude of the ultrasound signal depends on the angle between myocardial fibres and the ultrasound beam. ${ }^{26}$ Because systolic wall thickening depends not only on intrinsic myocyte contraction but also on the interstitial collagen matrix and fibre realignment within the ventricular wall, it is not surprising that incorporating end diastolic echo amplitude, which reflects collagen and fibre alignment, enhances the correlation between cyclic variation and geometric indices of systolic activity such as wall thickening. This helps to explain some of the anomalies reported previously, such as significant cyclic variation in the presence of virtually absent wall thickening and motion as observed in infarcted and peri-infarcted areas of myocardium. ${ }^{24}$ In that case end diastolic echo amplitude would be increased, resulting in a lower cyclic variation index compared with the observed absolute level of cyclic variation in decibels. However, as previous workers have shown and as this study confirms, the extent of cyclic variation is multifactorial and cannot be solely related to wall thickening. Of note in this study is the negative correlation between age and cyclic variation seen in the posterior wall of the ventricle which was not mirrored by a similar relation between age and systolic thickening. This is similar to that recently reported by Masuyama et al $^{30}$ and may reflect the many subtle changes in the myocardium which occur with aging, in particular the well documented increases in myocardial collagen. ${ }^{31} 32$

\section{LIMITATIONS}

There are several limitations to this study which must be considered. Although in this study subjects were generally good echo subjects, image quality as in most echocardiographic studies can be unsatisfactory. Full echo amplitude studies could not be analysed in three subjects. Also, owing to rotation and translation of the heart during the cardiac cycle, end systolic and end diastolic frames may not image directly comparable areas of myocardium. Simultaneous $M$ mode and colour encoded images could not be obtained with the system we used and this may also introduce a source of error. Additionally the well known inaccuracies of $M$ mode measurements particularly with respect to interventricular septal thickness must be considered. Finally, the present system still requires the master gain to be set using the parietal pericardial signals and this may introduce a small degree of subjectivity in overall values though not the extent of cyclic change.

\section{CONCLUSIONS}

Our observations in human subjects of a significant correlation between cyclic variation and systolic wall thickening strengthen the hypothesis that cyclic variation predominantly reflects local shortening within the myocardium. We have shown that there is a correlation between cyclic variation index and systolic wall thickening which is valid over a wide range of myocardial function and which is not altered by distorted wall motion. We also showed the importance of incorporating background echo amplitude into relations between cyclic variation in echo amplitude and systolic thickening. This emphasises the importance of having the capability to use in vivo standardisation which minimises the effect of the near field intervening tissue to enable relative levels of echo amplitude to be assigned. The clinical implications of this are that it may provide a noninvasive quantitative method for assessing segmental myocardial shortening which is sensitive to changes in contractility, local loading conditions, and local architecture. For example, this could be applied to determining the myocardium at risk during coronary angioplasty and the effect of interventions to limit this and the assessment of salvagable myocardium during thrombolytic therapy. In both, conventional geometric measurements of performance are limited by the distortion of wall motion in the peri-infarction or ischaemic zone. In these 
circumstances, tissue characterisation may provide more useful information on tissue viability and function.

In conclusion, this study provides evidence that cyclic variation in echo amplitude reflects segmental myocardial shortening in normal and abnormal hearts and this can be measured by analysis of grey scale images from studies acquired in real time.

DAL is a Junior Research Fellow of the British Heart Foundation.

1 Mimbs JW, O'Donnell M, Miller JG, Sobel BE. Changes in ultrasonic attenuation indicative of early myocardial ultrasonic attenuation indicative of early myocar

2 Logan-Sinclair R, Wong CM, Gibson DG. Clinical application of amplitude processing of echocardiographic images. Br Heart J 1981;45:621-7.

3 Parisi AF, Nieminen M, O'Boyle JE, et al. Enhanced detection of the evolution of tissue changes after acute myocardial infarction using color-encoded two-dimensional echocardiography. Circulation 1982;66:764-70.

4 Davies J, Gibson DG, Foale R, et al. Echocardiographic features of eosinophilic endomyocardial disease. Br Heart J 1982;48:434-40.

5 Shaw TRD, Logan-Sinclair RB, Surin C, et al. Relation between regional echo intensity and myocardial connective tissue in chronic left ventricular disease. $\mathrm{Br}$ Heart 1984;51:46-53.

6 Shapiro LM, Moore RB, Logan-Sinclair RB, Gibson DG Relation of regional echo amplitude to left ventricula function and the electrocardiogram in left ventricula hypertrophy. Br Heart J 1984;52:99-105.

7 Perez JE, Barzilai B, Madaras EI, et al. Applicability of ultrasonic tissue characterization for longitudinal assessultrasonic tissue characterization for longitudinal assess-
ment and differentiation of calcification and fibrosis in ment and differentiation of calcification and fibrosiomyopathy. J Am Coll Cardiol 1984;4:88-95.

8 Miller JG, Perez JE, Sobel BE. Ultrasonic characterization of myocardium. Prog Cardiovasc Dis 1985;28:85-110.

9 Vered Z, Barzilai B, Mohr GA, et al. Quantitative ultrasonic tissue characterization with real-time integrated backscatter imaging in normal human subjects and in patients with dilated cardiomyopathy. Circulation 1987;5:1067-73.

10 Lythall DA, Gibson DG, Swanson K, Mitchell AG, Ilsley CD, Yacoub MH. Quantitative analysis of myocardial CD, Yacoub MH. Quantitative analysis of myocardial echo amplitude-a useful marker of cardiac rejection
[abstract?. Circulation 1989;80(suppl II):677.

11 Madaras EI, Barzilai B, Perez JE, Sobel BE, Miller JG. Changes in myocardial backscatter throughout the cardia cycle. Ultrason Imaging 1983;5:229-39.

12 Olshansky B, Collins SM, Skorton DJ, Prasad NV. Variation of left ventricular myocardial gray level on twodimensional echocardiograms as a result of cardiac contraction. Circulation 1984;6:972-7.

13 Barzilai B, Madaras EI, Sobel BE, Miller JG, Perez JE. Effects of myocardial contraction on ultrasonic backscatter before and after ischemia. Am J Physiol 1984;247: H478-83.

14 Wickline SA, Thomas LJ III, Miller JG, Sobel BE, Perez JE. A relationship between ultrasonic integrated back sE. A relationship between ultrasonic integrated back scatter and myocard
15 Bamber JC, Daft C. Adaptive filtering for reduction of speckle in ultrasonic pulse-echo images. Ultrasonics 1986; 5:41-4.

16 Massay RJ, Logan-Sinclair RB, Bamber JC, Gibson DG Quantitative effects of speckle reduction on cross sectional echocardiographic images. Br Heart J 1989;62:298-304.

17 Corya BC, Rasmussen S, Feigenbaum H, Knoebel SB, Black MJ. Systolic thickening and thinning of the septum and posterior wall in patients with coronary artery disease, congestive cardiomyopathy, and atrial septal defect. Circulation 1976;55(1):109-14

18 Wickline SA, Thomas LJ III, Miller JG, Sobel BE, Perez $\mathrm{JE}$. The dependence of myocardial ultrasonic integrated backscatter on contractile performance. Circulation 1985; 72(1):183-92.

19 Glueck RM, Mottley JC, Miller JG, Sobel BE, Sobel JE. Effects of coronary artery occlusion and reperfusion on cardiac-cycle dependent variation of myocardial ultrasonic backscatter. Circ Res 1985;56:683-9.

20 Wickline SA, Thomas LJ III, Miller JG, Sobel BE, Perez JE. Sensitive detection of the effects of reperfusion on myocardium by ultrasonic tissue characterization with integrated backscatter. Circulation 1986;74(2):389-400.

21 Zhao M, Zhang H, Robinson TF, Factor SM, Sonnenblick EH, Eng C. Profound structural alterations of the extracellular collagen matrix in postischemic dysfuncextracellular collagen matrix in postischemic dysfunc-
tional ("stunned") but viable myocardium. J Am Coll tional ("stunned") but

22 Sagar KB, Pelc LE, Rhyne TL, Wann LS, Waltier DC. Influence of heart rate, preload, afterload, and inotropic state on myocardial ultrasonic backscatter. Circulation 1988;77(2):478-83.

23 Hajduczki I, Jaffe M, Areeda J, et al. Ultrasonic backscatter and $2 \mathrm{D}$ echocardiographic wall motion during PTCA [abstract]. Circulation 1988;78(suppl II):442.

24 Milunski MR, Mohr GA, Perez JE, et al. Ultrasonic tissue characterization with integrated backscatter: acute myocardial ischemia, reperfusion, and stunned myocardium in patients. Circulation 1989;80:491-503.

25 Milunski MR, Canter CE, Wickline SA, Sobel BE, Miller JG, Perez JE. Cardiac cycle dependent variation of integrated backscatter is not distorted by abnormal myocardial wall motion in human subjects with paradoxical cardial wall motion in human subjects with paradoxical
septal motion. Ultrasound Med Biol 1989;15(4):311-7.

26 Aygen M, Popp RL. Influence of the orientation of myocardial fibres on echocardiographic images. Am J Cardiol dial fibres on ech

27 Greenbaum RA, Ho SY, Gibson DG, Becker AE, Anderson $\mathrm{RH}$. Left ventricular fibre architecture in man. Br Heart $J$ $1981 ; 45: 248-63$

28 Streeter DD Jr, Spotnitz HM, Patel DP, Ross J Jr, Sonnenblick EH. Fibre orientation in the canine left ventricle during diastole and systole. Circ Res 1969; 24:339-47.

29 Masuyama T, St Goar FG, Tye TL, Oppenheim G, Schnittger I, Popp RL. Ultrasonic tissue characterization of human hypertrophied hearts in vivo with cardiac cycledependent variation in integrated backscatter. Circulation 1989;80:925-34.

30 Masuyama T, Nellessen U, Schnittger I, Tye TL, Haskell WL, Popp RL. Ultrasonic tissue characterization with a real time integrated backscatter imaging system in normal and aging human hearts. J Am Coll Cardiol 1989;14: and aging

31 Lenkiewicz JE, Davies MJ, Rosen D. Collagen in human myocardium as a function of age. Cardiovasc Res 1972; 6:549-55.

32 Weber KT. Cardiac interstitium in health and disease: the fibrillar collagen network. J Am Coll Cardiol 1989; 13(7):1637-52. 